\title{
Atividade antimicrobiana in vitro dos extratos aquosos, hidroalcoólicos e alcoólicos das folhas de espécies da família Myrtaceae frente à cepas de bactérias de interesse
}

\author{
Antimicrobial activity in vitro of aqueous extracts, hydroalcoholic and spirits of \\ Myrtaceae family species leaves front of the strains of interest of bacteria
}

\author{
Francisca Hérica Chaves Albuquerque ${ }^{1}$, Karina da Silva Soares², Maria Auxiliadora Silva Oliveira ${ }^{3 *}$ \\ ${ }^{1}$ Farmacêutica, Centro Universitário UNINTA; ${ }^{2}$ Graduada em Saneamento Ambiental, Laboratório de Microbiologia
}

(Centro Universitário UNINTA); ${ }^{3}$ Professora do Centro Universitário UNINTA

\begin{abstract}
Resumo
Introdução: a Organização Mundial da Saúde (OMS) tem incentivado o estudo de plantas tradicionalmente conhecidas como medicinais, tendo como objetivo avaliar cientificamente os benefícios da utilização de medicamentos fitoterápicos e de conhecer, ao mesmo tempo, a segurança de seu uso indevido. Objetivos: este trabalho teve como objetivo avaliar a atividade antimicrobiana do extrato aquoso, hidroalcoólico e alcoólico de folhas de espécies da família Myrtaceae frente às cepas de bactérias de interesse. Metodologia: a análise da atividade antibacteriana dos extratos das folhas das plantas, jambo, azeitona preta, pitanga e goiaba foram verificados a partir do teste de difusão em Ágar Mueller-Hinton. As cepas utilizadas, após reativação, foram diluídas em solução salina estéril a $0,85 \%$. Sendo consideradas com potencial antimicrobiano aqueles extratos que geraram halos $\geq 7 \mathrm{~mm}$ (sete milímetros). Resultados: os resultados encontrados na análise dos dados do extrato aquoso demonstraram que Eugenia uniflora, Syzygium cumini e Psidium guajava apresentaram atividade antimicrobiana sobre Staphylococcus aureus, na concentração de $100 \mathrm{mg} / \mathrm{mL}$. Na análise dos dados dos extratos hidroalcoólico, Syzygium cumini demonstrou atividade antimicrobiana na concentração de $100 \mathrm{mg} / \mathrm{mL}$ sobre Pseudomonas aeruginosa, diante de Staphylococcus aureus, Eugenia uniflora, Syzygium malaccense, Syzygium cumini e Psidium guajava demonstraram atividade na concentração de $100 \mathrm{mg} / \mathrm{mL}$ cada. Observando os resultados dos extratos alcoólicos, Psidium guajava e Syzygium cumini demonstraram atividade antimicrobiana diante das quatro cepas estudadas. Conclusão: o presente estudo demonstrou que os extratos alcoólicos das espécies em estudo apresentaram melhor atividade antimicrobiana e maior espectro de ação quando comparado ao extrato aquoso e hidroalcoólico.
\end{abstract}

Palavras-chave: Myrtaceae. Antibiose. Plantas Medicinais.

\begin{abstract}
Introduction: this study aimed to evaluate the antimicrobial activity of aqueous extract, hydro-alcoholic and alcoholic leaf front Myrtaceae family species to strains of bacteria of interest. Objective: this study aimed to evaluate the antimicrobial activity of aqueous extract, hydro alcoholic and alcoholic leaves Myrtaceae front family species to strains of bacteria of interest. Methodology: the antibacterial activity of plant leaves extracts, rose apple, black olive, cherry and guava were verified from the diffusion test on Mueller-Hinton agar. Where the strains used after reactivation were diluted in $0.85 \%$ sterile saline. Being considered with antimicrobial potential that statement that generated halos $\geq 7 \mathrm{~mm}$ (seven millimeters). Results: the results of the analysis of the aqueous extract data demonstrated that Eugenia uniflora, Syzygium cumini and Psidium guajava showed antimicrobial activity against Staphylococcus aureus at a concentration of $100 \mathrm{mg} / \mathrm{mL}$. In the data analysis of hydro-alcoholic extracts, Syzygium cumini demonstrated antimicrobial activity at a concentration of $100 \mathrm{mg} / \mathrm{mL}$ of Pseudomonas aeruginosa, before Staphylococcus aureus, Eugenia uniflora, Syzygium malaccense, Syzygium cumini and Psidium guajava showed activity at a concentration of $100 \mathrm{mg} / \mathrm{mL}$ each. Noting the results of alcoholic extracts, Psidium guajava and Syzygium cumini demonstrated antimicrobial activity against the four strains studied. Conclusion: the present study demonstrated that the alcoholic extracts of the species under study presented better antimicrobial activity and a greater spectrum of action when compared to the aqueous and hydroalcoholic extract.

Keyword: Myrtaceae. Antibiosis. Plants Medicinal.
\end{abstract}

\section{INTRODUÇÃO}

O uso de plantas medicinais na recuperação da saúde tem evoluído ao longo dos tempos desde as formas mais simples de tratamento local, provavelmente utilizadas pelo homem das cavernas, até as formas tecnologica-

Correspondente/Corresponding: *Maria Auxiliadora Silva Oliveira End: R. Cel. Antonio Rodrigues Magalhães, Bairro D. Expedito Lopes, 359, Sobral-CE - Tel: (88) 3112-3500-E-mail: ecobio@zipmail.com.br mente sofisticadas das indústrias farmacêuticas, nos dias modernos. Diante das enormes diferenças entre as duas maneiras de uso, há um fato comum entre elas: nos dois casos o homem percebeu a presença da existência de algo que, administrado sob a forma de mistura complexa como nos chás, garrafadas, tinturas, pós, etc., ou como substância pura isolada e transformado em comprimidos, gotas, pomadas ou cápsulas, têm a propriedade de provocar reações benéficas no organismo, capazes de resultar 
na recuperação da saúde e cooperar para melhoria da qualidade de vida (LORENZI; MATOS, 2008).

A Organização Mundial da Saúde (OMS), desde 1977, tem incentivado o estudo de plantas tradicionalmente conhecidas como medicinais, tendo como objetivo avaliar cientificamente os benefícios da utilização de medicamentos fitoterápicos e de conhecer, ao mesmo tempo, a segurança de seu uso indevido (YUNES; CALIXTO, 2001).

O foco deste estudo são quatro espécies da família Myrtaceae, pertencente à ordem Myrtales, a qual é constituída por cerca de 132 gêneros e mais de 5.600 espécies. No Brasil, é composta aproximadamente por 24 gêneros e 927 espécies (SOBRAL, 2003). Myrtaceae é dividida em duas subfamílias: Myrtoideae apresenta frequentemente frutos em bagas, onde inclui os gêneros Myrtus, Psidium, Pimenta, Eugenia, Pseudocaryophyllus e Syzygium, e Leptospermoideae. Os frutos, em sua maioria apresentam semente única do tipo noz, à qual pertencem os gêneros Eucalyptus, Leptospermum e Malaleuca (TYLER, 1999). A família Myrtaceae possui espécies com atividades antioxidante, hipoglicemiante, antireumática, sendo também utilizadas em distúrbios estomacais e como anti-hipertensivas (LORENZI; MATOS, 2008).

A procura por alternativas terapêuticas complementares à farmacoterapia tradicional converteu-se recentemente numa diretriz importante no contexto da saúde pública. Atualmente, a pesquisa com plantas medicinais é a base fundamental nas políticas de saúde implementadas pelas esferas governamentais como parte da Política Nacional de Práticas Integrativas e Complementares do Sistema Único de Saúde (BRASIL, 2006).

Embora exista uma grande diversidade de antibióticos que agem sobre diversos microrganismos patogênicos, estudos procuram por um antibiótico ideal, ou seja, aquele que apresenta maior espectro de ação, menor custo, menor toxicidade, e menor indício de resistência bacteriana, em vista que já existe resistência bacteriana a alguns produtos antimicrobianos (PAZHANI et al., 2004). Essa atividade antibacteriana desejada pode ser encontrada em espécies de plantas medicinais. Em uma população com baixo acesso a medicamentos, como a brasileira, agregar garantias científicas a essa prática terapêutica traz variadas vantagens (PAZHANI et al., 2004).

A flora brasileira é altamente diversificada em espécies que na sua maioria ainda não foram pesquisadas cientificamente quanto à sua atividade antibacteriana. Diante desta realidade, estudar tratamentos alternativos ou complementares aos antibióticos tradicionais, tais como são os produtos naturais, com a perspectiva de utilizar estas plantas como ativo de novas formulações motivaram a execução da referida pesquisa.

A presente investigação tem como objetivo avaliar a atividade antimicrobiana do extrato aquoso, hidroalcoólico e alcoólico de folhas de espécies da família Myrtaceae frente às cepas de interesse.

\section{METODOLOGIA}

A pesquisa foi realizada no Núcleo de Bioprospecção e Experimentação Molecular Aplicada (NUBEM - INTA), localizado no Centro Universitário UNINTA, na cidade de Sobral-CE.

A coleta das folhas sadias e ramos com flores da Eugenia uniflora (pitanga), Syzygium malaccense (jambo-vermeIho), Syzygium cumini (azeitona preta) e Psidium guajava (goiaba) foram realizadas na cidade de Alcântaras-Ce. Exemplares das plantas foram utilizadas para confecção de exsicatas, onde foram identificadas no herbário Prof. José de Abreu Matos da Universidade Estadual Vale do AcaraúUVA, no município de Sobral -CE e estão depositadas sob o № 18852 (Eugenia uniflora), 18851 (Syzigium malaccense), 18850 (Syzigium cumini), 18849 (Psidium guajava).

Depois de coletadas, as folhas foram lavadas e secadas à sombra e sem sobreposição, por período de cinco dias (MATOS, 2007). Em seguida, foram removidos os pecíolos e pulverizadas. Usou-se $3 \mathrm{~g}$ de pó e $30 \mathrm{~mL}$ de água destilada estéril, para cada planta, através do método de infusão, depois do resfriamento, foram mantidas na geladeira em reservatórios protegidos da luz. Para o extrato hidroalcoólico foi adicionado $3 \mathrm{~g}$ do pó em $30 \mathrm{~mL}$ da solução contendo água destilada estéril com álcool etílico 100\% PA (1:1 v/v). Para o extrato alcoólico foi usado $3 \mathrm{~g}$ do pó em $30 \mathrm{~mL}$ de álcool PA. Após 72 horas em maceração, as soluções foram filtradas em papel-filtro adquirindo-se, dessa forma, os extratos brutos.

As diluições para os testes foram feitas a partir do extrato bruto. Foram testados para todas as espécies 03 diluições: $100 \mathrm{mg} / \mathrm{mL}, 50 \mathrm{mg} / \mathrm{mL}$ e $25 \mathrm{mg} / \mathrm{mL}$. Estes foram diluídos em reservatórios estéreis e protegidos da luz. Como controle negativo, no extrato aquoso, foi utilizado água destilada estéril; no extrato hidroalcoólico foi utilizada solução contendo água destilada estéril com álcool etílico $100 \%$ PA (1:1 v/v) e no extrato alcoólico foi utilizado álcool etílico $100 \%$.

As bactérias utilizadas foram: Streptococous pyogenes (ATCC19615), Pseudomonas aeruginosa (ATCC15442), Enterococcus faecalis (ATCC4083), Staphylococus aureus (ATCC6530). As mesmas encontravam-se armazenadas em Brain Heart Infusion (BHI) glicerol $20 \%$ em freezer a estão preservadas no Núcleo de Bioprospecção e Experimentação Molecular Aplicada (NUBEM), Sobral-Ce.

Para análise da atividade antimicrobiana dos extratos das folhas das plantas pitanga, azeitona-preta, jambo e goiaba, foram realizadas em triplicata e verificadas a partir do teste de difusão em Ágar Mueller-Hinton. Todas as cepas, após reativação, foram diluídas em solução salina estéril a 0,85\% e ajustada de acordo com a escala padrão de McFarland 0,5\% (TAVEIRA, 2007). Como controle negativo, no extrato aquoso, foi utilizado água destilada estéril; no extrato hidroalcoólico foi utilizada solução contendo água destilada estéril com álcool etílico 100\% PA (1:1 v/v) e no extrato alcoólico foi utilizado álcool etílico $100 \%$. Como controle positivo foram utilizados discos contendo $10 \mu \mathrm{g}$ de Cefuroxima. 
Foram recolhidas alíquotas das culturas bacterianas previamente diluídas e semeadas, com auxílio de um $s w a b$, pela técnica do esgotamento, em placas de petri contendo Ágar Mueller-Hinton.

As placas foram incubadas em estufa a $37^{\circ} \mathrm{C}$ por um período de 24 horas. Após o período de incubação, mediram-se os halos de inibição com auxílio de uma régua. Foram considerados com potencial antimicrobiano contra os isolados bacterianos aqueles extratos que geraram halos $\geq 7 \mathrm{~mm}$ (sete milímetros) (ARAUJO et al., 2011).

\section{RESULTADOS}

$\mathrm{Na}$ análise dos resultados dos extratos aquosos, Eugenia uniflora, Syzygium cumini e Psidium guajava demonstraram atividade antimicrobiana sobre Staphylococcus aureus com halos de $12 \mathrm{~mm}, 8 \mathrm{~mm}$ e $7 \mathrm{~mm}$ na concentração de $100 \mathrm{mg} / \mathrm{mL}$, respectivamente. $\mathrm{Na}$ tabela 1 estão sumarizados os resultados dos ensaios de antibiose com extrato aquoso.

Tabela 1 - Atividade antimicrobiana de extratos aquosos de folhas de Eugenia uniflora, Syzygium malaccense, Syzygium cumini e Psidium guajava nas concentrações de $25 \mathrm{mg} / \mathrm{mL}, 50 \mathrm{mg} / \mathrm{mL}$ e $100 \mathrm{mg} / \mathrm{mL}$.

\begin{tabular}{|c|c|c|c|c|c|}
\hline \multicolumn{4}{|c|}{ Concentrações do extrato aquoso (mg/mL) } & \multirow[t]{2}{*}{$\mathrm{CN}$} & \multirow[t]{2}{*}{$\mathrm{CP}$} \\
\hline \multicolumn{4}{|c|}{ Sthaphylococcus aureus } & & \\
\hline & $25 \mathrm{mg} / \mathrm{mL}$ & $50 \mathrm{mg} / \mathrm{mL}$ & $100 \mathrm{mg} / \mathrm{mL}$ & & \\
\hline Eugenia uniflora & $10 \mathrm{~mm}$ & $12 \mathrm{~mm}$ & $12 \mathrm{~mm}$ & $0 \mathrm{~mm}$ & $23 \mathrm{~mm}$ \\
\hline Syzygium malaccense & $0 \mathrm{~mm}$ & $0 \mathrm{~mm}$ & $0 \mathrm{~mm}$ & $0 \mathrm{~mm}$ & $22 \mathrm{~mm}$ \\
\hline Syzygium cumini & $0 \mathrm{~mm}$ & $0 \mathrm{~mm}$ & $8 \mathrm{~mm}$ & $0 \mathrm{~mm}$ & $25 \mathrm{~mm}$ \\
\hline Psidium guajava & $0 \mathrm{~mm}$ & $0 \mathrm{~mm}$ & $7 \mathrm{~mm}$ & $0 \mathrm{~mm}$ & $24 \mathrm{~mm}$ \\
\hline \multicolumn{6}{|c|}{ Streptococcus pyogenes } \\
\hline Eugenia uniflora & $0 \mathrm{~mm}$ & $0 \mathrm{~mm}$ & $0 \mathrm{~mm}$ & $0 \mathrm{~mm}$ & $14 \mathrm{~mm}$ \\
\hline Syzygium malaccense & $0 \mathrm{~mm}$ & $0 \mathrm{~mm}$ & $0 \mathrm{~mm}$ & $0 \mathrm{~mm}$ & $17 \mathrm{~mm}$ \\
\hline Syzygium cumini & $0 \mathrm{~mm}$ & $0 \mathrm{~mm}$ & $0 \mathrm{~mm}$ & $0 \mathrm{~mm}$ & $15 \mathrm{~mm}$ \\
\hline Psidium guajava & $0 \mathrm{~mm}$ & $0 \mathrm{~mm}$ & $0 \mathrm{~mm}$ & $0 \mathrm{~mm}$ & $14 \mathrm{~mm}$ \\
\hline \multicolumn{6}{|c|}{ Enterecoccus faecalis } \\
\hline Eugenia uniflora & $0 \mathrm{~mm}$ & $0 \mathrm{~mm}$ & $0 \mathrm{~mm}$ & $0 \mathrm{~mm}$ & $13 \mathrm{~mm}$ \\
\hline Syzygium malaccense & $0 \mathrm{~mm}$ & $0 \mathrm{~mm}$ & $0 \mathrm{~mm}$ & $0 \mathrm{~mm}$ & $12 \mathrm{~mm}$ \\
\hline Syzygium cumini & $0 \mathrm{~mm}$ & $0 \mathrm{~mm}$ & $0 \mathrm{~mm}$ & $0 \mathrm{~mm}$ & $14 \mathrm{~mm}$ \\
\hline Psidium guajava & $0 \mathrm{~mm}$ & $0 \mathrm{~mm}$ & $0 \mathrm{~mm}$ & $0 \mathrm{~mm}$ & $18 \mathrm{~mm}$ \\
\hline \multicolumn{6}{|c|}{ Pseudomonas aeruginosa } \\
\hline Eugenia uniflora & $0 \mathrm{~mm}$ & $0 \mathrm{~mm}$ & $0 \mathrm{~mm}$ & $0 \mathrm{~mm}$ & $12 \mathrm{~mm}$ \\
\hline Syzygium malaccense & $0 \mathrm{~mm}$ & $0 \mathrm{~mm}$ & $0 \mathrm{~mm}$ & $0 \mathrm{~mm}$ & $12 \mathrm{~mm}$ \\
\hline Syzygium cumini & $0 \mathrm{~mm}$ & $0 \mathrm{~mm}$ & $0 \mathrm{~mm}$ & $0 \mathrm{~mm}$ & $13 \mathrm{~mm}$ \\
\hline Psidium guajava & $0 \mathrm{~mm}$ & $0 \mathrm{~mm}$ & $0 \mathrm{~mm}$ & $0 \mathrm{~mm}$ & $11 \mathrm{~mm}$ \\
\hline
\end{tabular}

* Controle positivo (CP): Cefuroxima. Controle negativo (CN): Água destilada estéril.

Fonte: Autoria própria

$\mathrm{Na}$ análise dos dados dos extratos hidroalcoólicos, Syzygium cumini demonstrou atividade antimicrobiana com halo de $7 \mathrm{~mm}$ na concentração de $100 \mathrm{mg} / \mathrm{mL}$ sobre Pseudomonas aeruginosa. Porém, não foi observada atividade antimicrobiana das plantas estudadas sobre Streptococcus pyogenes e Enterecoccus faecalis. Na tabela 2 estão sumarizados os resultados dos ensaios de antibiose com extrato hidroalcoólico.

Tabela 2 - Atividade antimicrobiana de extratos hidroalcoólicos de folhas de Eugenia uniflora, Syzygium malaccense, Syzygium cumini e Psidium guajava nas concentrações de $25 \mathrm{mg} / \mathrm{mL}, 50 \mathrm{mg} / \mathrm{mL}$ e $100 \mathrm{mg} / \mathrm{mL}$.

\begin{tabular}{|c|c|c|c|c|c|}
\hline Extrato & \multicolumn{3}{|c|}{ Concentrações do extrato hidroalcoólico (mg/mL) } & \multirow[t]{2}{*}{ CN } & \multirow[t]{2}{*}{$\mathrm{CP}$} \\
\hline \multicolumn{4}{|c|}{ Staphylococcus aureus } & & \\
\hline & $25 \mathrm{mg} / \mathrm{mL}$ & $50 \mathrm{mg} / \mathrm{mL}$ & $100 \mathrm{mg} / \mathrm{mL}$ & & \\
\hline Eugenia uniflora & $12 \mathrm{~mm}$ & $12 \mathrm{~mm}$ & $14 \mathrm{~mm}$ & $0 \mathrm{~mm}$ & $20 \mathrm{~mm}$ \\
\hline Syzygium malaccense & $0 \mathrm{~mm}$ & $0 \mathrm{~mm}$ & $7 \mathrm{~mm}$ & $0 \mathrm{~mm}$ & $16 \mathrm{~mm}$ \\
\hline Syzygium cumini & $7 \mathrm{~mm}$ & $8 \mathrm{~mm}$ & $10 \mathrm{~mm}$ & $0 \mathrm{~mm}$ & $20 \mathrm{~mm}$ \\
\hline Psidium guajava & $7 \mathrm{~mm}$ & $8 \mathrm{~mm}$ & $9 \mathrm{~mm}$ & $0 \mathrm{~mm}$ & $22 \mathrm{~mm}$ \\
\hline \multicolumn{6}{|c|}{ Streptococcus pyogenes } \\
\hline Eugenia uniflora & $0 \mathrm{~mm}$ & $0 \mathrm{~mm}$ & $0 \mathrm{~mm}$ & $0 \mathrm{~mm}$ & $13 \mathrm{~mm}$ \\
\hline Syzygium malaccense & $0 \mathrm{~mm}$ & $0 \mathrm{~mm}$ & $0 \mathrm{~mm}$ & $0 \mathrm{~mm}$ & $13 \mathrm{~mm}$ \\
\hline
\end{tabular}


Francisca Hérica Chaves Albuquerque, Karina da Silva Soares, Maria Auxiliadora Silva Oliveira

\begin{tabular}{|c|c|c|c|c|c|}
\hline Extrato & \multicolumn{3}{|c|}{ Concentrações do extrato hidroalcoólico (mg/mL) } & $\mathrm{CN}$ & $\mathrm{CP}$ \\
\hline \multicolumn{6}{|c|}{ Staphylococcus aureus } \\
\hline & $25 \mathrm{mg} / \mathrm{mL}$ & $50 \mathrm{mg} / \mathrm{mL}$ & $100 \mathrm{mg} / \mathrm{mL}$ & & \\
\hline Syzygium cumini & $0 \mathrm{~mm}$ & $0 \mathrm{~mm}$ & $0 \mathrm{~mm}$ & $0 \mathrm{~mm}$ & $14 \mathrm{~mm}$ \\
\hline Psidium guajava & $0 \mathrm{~mm}$ & $0 \mathrm{~mm}$ & $0 \mathrm{~mm}$ & $0 \mathrm{~mm}$ & $14 \mathrm{~mm}$ \\
\hline \multicolumn{6}{|c|}{ Enterecoccus faecalis } \\
\hline Eugenia uniflora & $0 \mathrm{~mm}$ & $0 \mathrm{~mm}$ & $0 \mathrm{~mm}$ & $0 \mathrm{~mm}$ & $12 \mathrm{~mm}$ \\
\hline Syzygium malaccense & $0 \mathrm{~mm}$ & $0 \mathrm{~mm}$ & $0 \mathrm{~mm}$ & $0 \mathrm{~mm}$ & $13 \mathrm{~mm}$ \\
\hline Syzygium cumini & $0 \mathrm{~mm}$ & $0 \mathrm{~mm}$ & $0 \mathrm{~mm}$ & $0 \mathrm{~mm}$ & $10 \mathrm{~mm}$ \\
\hline Psidium guajava & $0 \mathrm{~mm}$ & $0 \mathrm{~mm}$ & $0 \mathrm{~mm}$ & $0 \mathrm{~mm}$ & $10 \mathrm{~mm}$ \\
\hline \multicolumn{6}{|c|}{ Pseudomonas aeruginosa } \\
\hline Eugenia uniflora & $0 \mathrm{~mm}$ & $0 \mathrm{~mm}$ & $0 \mathrm{~mm}$ & $0 \mathrm{~mm}$ & $11 \mathrm{~mm}$ \\
\hline Syzygium malaccense & $0 \mathrm{~mm}$ & $0 \mathrm{~mm}$ & $0 \mathrm{~mm}$ & $0 \mathrm{~mm}$ & $11 \mathrm{~mm}$ \\
\hline Syzygium cumini & $0 \mathrm{~mm}$ & $0 \mathrm{~mm}$ & $0 \mathrm{~mm}$ & $0 \mathrm{~mm}$ & $11 \mathrm{~mm}$ \\
\hline Psidium guajava & $0 \mathrm{~mm}$ & $0 \mathrm{~mm}$ & $0 \mathrm{~mm}$ & $0 \mathrm{~mm}$ & $11 \mathrm{~mm}$ \\
\hline
\end{tabular}

* Controle positivo (CP): Cefuroxima. Controle negativo (CN): Solução contendo água destilada estéril com álcool etílico 100\% PA (1:1 v/v).

Fonte: Autoria própria

E sobre Staphylococcus aureus, Eugenia uniflora, Psidium guajava e Syzygium cumini demonstraram atividade com halos de $11 \mathrm{~mm}, 8 \mathrm{~mm}$ e $10 \mathrm{~mm}$ respectivamente, na concentração de $100 \mathrm{mg} / \mathrm{mL}$ cada. O extrato alcoólico de Syzygium malaccense não apresentou atividade antimicrobiana frente às cepas estudadas. Na tabela 3 estão sumarizados os resultados dos ensaios de antibiose com os extratos alcoólicos.

Tabela 3 - Atividade antimicrobiana de extratos alcoólicos de folhas de Eugenia uniflora, Syzygium malaccense, Syzygium cumini e Psidium guajava nas concentrações de $25 \mathrm{mg} / \mathrm{mL}, 50 \mathrm{mg} / \mathrm{mL}$ e $100 \mathrm{mg} / \mathrm{mL}$.

\begin{tabular}{|c|c|c|c|c|c|}
\hline Extrato & \multicolumn{3}{|c|}{ Concentrações do extrato alcoólico (mg/mL) } & CN & $\mathrm{CP}$ \\
\hline \multicolumn{6}{|c|}{ Staphylococcus aureus } \\
\hline & $25 \mathrm{mg} / \mathrm{mL}$ & $50 \mathrm{mg} / \mathrm{mL}$ & $100 \mathrm{mg} / \mathrm{mL}$ & & \\
\hline Eugenia uniflora & $8 \mathrm{~mm}$ & $10 \mathrm{~mm}$ & $11 \mathrm{~mm}$ & $0 \mathrm{~mm}$ & $23 \mathrm{~mm}$ \\
\hline Syzygium malaccense & $0 \mathrm{~mm}$ & $0 \mathrm{~mm}$ & $0 \mathrm{~mm}$ & $0 \mathrm{~mm}$ & $18 \mathrm{~mm}$ \\
\hline Syzygium cumini & $7 \mathrm{~mm}$ & $8 \mathrm{~mm}$ & $10 \mathrm{~mm}$ & $0 \mathrm{~mm}$ & $24 \mathrm{~mm}$ \\
\hline Psidium guajava & $0 \mathrm{~mm}$ & $7 \mathrm{~mm}$ & $8 \mathrm{~mm}$ & $0 \mathrm{~mm}$ & $18 \mathrm{~mm}$ \\
\hline \multicolumn{6}{|c|}{ Streptococcus pyogenes } \\
\hline Eugenia uniflora & $0 \mathrm{~mm}$ & $0 \mathrm{~mm}$ & $0 \mathrm{~mm}$ & $0 \mathrm{~mm}$ & $13 \mathrm{~mm}$ \\
\hline Syzygium malaccense & $0 \mathrm{~mm}$ & $0 \mathrm{~mm}$ & $0 \mathrm{~mm}$ & $0 \mathrm{~mm}$ & $14 \mathrm{~mm}$ \\
\hline Syzygium cumini & $7 \mathrm{~mm}$ & $9 \mathrm{~mm}$ & $11 \mathrm{~mm}$ & $0 \mathrm{~mm}$ & $13 \mathrm{~mm}$ \\
\hline Psidium guajava & $0 \mathrm{~mm}$ & $7 \mathrm{~mm}$ & $10 \mathrm{~mm}$ & $0 \mathrm{~mm}$ & $24 \mathrm{~mm}$ \\
\hline \multicolumn{6}{|c|}{ Enterecoccus faecalis } \\
\hline Eugenia uniflora & $0 \mathrm{~mm}$ & $0 \mathrm{~mm}$ & $0 \mathrm{~mm}$ & $0 \mathrm{~mm}$ & $12 \mathrm{~mm}$ \\
\hline Syzygium malaccense & $0 \mathrm{~mm}$ & $0 \mathrm{~mm}$ & $0 \mathrm{~mm}$ & $0 \mathrm{~mm}$ & $10 \mathrm{~mm}$ \\
\hline Syzygium cumini & $8 \mathrm{~mm}$ & $10 \mathrm{~mm}$ & $11 \mathrm{~mm}$ & $0 \mathrm{~mm}$ & $13 \mathrm{~mm}$ \\
\hline Psidium guajava & $0 \mathrm{~mm}$ & $7 \mathrm{~mm}$ & $8 \mathrm{~mm}$ & $0 \mathrm{~mm}$ & $13 \mathrm{~mm}$ \\
\hline \multicolumn{6}{|c|}{ Pseudomonas aeruginosa } \\
\hline Eugenia uniflora & $0 \mathrm{~mm}$ & $0 \mathrm{~mm}$ & $0 \mathrm{~mm}$ & $0 \mathrm{~mm}$ & $11 \mathrm{~mm}$ \\
\hline Syzygium malaccense & $0 \mathrm{~mm}$ & $0 \mathrm{~mm}$ & $0 \mathrm{~mm}$ & $0 \mathrm{~mm}$ & $12 \mathrm{~mm}$ \\
\hline Syzygium cumini & $7 \mathrm{~mm}$ & $8 \mathrm{~mm}$ & $9 \mathrm{~mm}$ & $0 \mathrm{~mm}$ & $12 \mathrm{~mm}$ \\
\hline Psidium guajava & $0 \mathrm{~mm}$ & $7 \mathrm{~mm}$ & $8 \mathrm{~mm}$ & $0 \mathrm{~mm}$ & $10 \mathrm{~mm}$ \\
\hline
\end{tabular}

* Controle positivo (CP): Cefuroxima. Controle negativo (CN): álcool etílico 100\% PA.

Fonte: Autoria própria

\section{DISCUSSÃO}

Ao tentar comparar estudos com plantas é incontestada a dificuldade de avaliação entre os resultados em consequência da atuação das diversas variáveis que vão desde os aspectos relativos ao solo e climáticos, que influenciam na composição química, como também o estágio do desenvolvimento do vegetal, forma da coleta, parte da planta a ser estudada, forma de preparo do material para estudo, altitude, tipo de solo, mudanças externas bem como situação de estresse, como queimaduras e enchentes também podem interferir na produção de compostos químicos (ALVES; TRESMONDI; LONGUI, 2008; AURICCHIO; BACCHI, 2003).

Ao comparar os resultados das respostas inibitórias (diâmetro do halo) obtidas nesse estudo com a literatura, verifica-se que as plantas pesquisadas apresentaram res- 
postas distintas com relação à atividade antimicrobiana. Para Lopes et al. (2006) a formação de halos de inibição sob os microrganismos testados é devido a um efeito sinérgico de todos os seus constituintes, compostos fitoquímicos: tanino, fenóis, flavonóides e alcalóides.

Conforme resultados encontrados por Furtado et al. (2015), verificaram que S. aureus mostrou-se sensível ao extrato aquoso de Eucalyptus globulus (Myrtaceae), da mesma forma que ao analisar a Tabela 1, evidencia-se essa espécies de bactéria sendo afetada por pelo menos uma espécie vegetal (Eugenia uniflora) também em extrato aquoso. Eller et al. (2015), afirmam que o resultado positivo sobre bactérias gram-positivas é mais comum de se verificar, pois estas apresentam mais sensibilidade frente a metabólitos vegetais. A afirmação destes autores confirmam os resultados encontrados neste experimento.

Testando extratos aquosos de Psidium guajava, Menezes, Souza e Botelho (2004) observaram resultados positivos para atividade antimicrobiana, com halos de inibição em média de $25 \mathrm{~mm}$ para Sthaphylococcus aureus. Esse resultado confirma os obtidos nesse estudo, mas há divergência no tamanho dos halos, que pode ser justificada pela metodologia aplicada na obtenção dos extratos ou na concentração na qual foi utilizada.

Gonçalves et al. (2008) observaram que Streptococcus pyogenes, Proteus mirabilis e Staphylococcus aureus apresentaram sensibilidade contra o extrato de Psidium guajava, não sendo constatada ação contra Escherichia coli e Pseudomonas aeruginosa. A divergência de resultados para algumas cepas pode ser justificada pela metodologia aplicada na obtenção dos extratos que sugerem possuir diferentes classes biossintéticas e químicas.

Sobre a espécie vegetal Eugenia uniflora, investigada neste experimento, muitos autores apontam sua atividade antimicrobiana contra S. aureus, Salmonella choleraesuis, Pseudomonas aeruginosa e Candida albicans, reforçando que esta planta tem potencial bioprospector (AURICCHIO et al., 2007; BOUZADA et al., 2007; COSTA et al., 2005; OLIVEIRA; SOARES; PADILHA, 2007).

Ao confrontar os resultados das respostas inibitórias (diâmetro do halo) obtidas neste estudo com a literatura, verifica-se que a Eugenia uniflora apresenta respostas distintas com relação à atividade antimicrobiana, no extrato alcoólico deste estudo a espécie apresentou halos somente contra Sthaphylococcus aureus.

É interessante notar que o extrato da Syzygium cumini apresenta atividade antimicrobiana frente às bactérias Gram-positiva (Staphylococcus aureus) e Gram-negativa (Pseudomonas aeruginosa), as quais são diferentes quanto à estrutura de sua membrana (SILVA, 2010). Geralmente bactérias Gram-negativas apresentam resistência aos antibióticos devido à diminuição da permeabilidade que ocorre em sua membrana externa (MOREIRA; FREIRE, 2011). Os resultados sugerem que flavonóides, saponinas e alcalóides, podem estar contribuindo para a atividade antimicrobiana observada contra as cepas Gram-negativas e, especialmente, contra as cepas Gram-positivas.
Lima et al. (2012), testando o extrato hidroalcoólico das folhas de Syzygium cumini na avaliação antimicrobiana, utilizando o método da difusão em ágar - poço observou atividade antibacteriana em relação a Staphylococcus aureus, Escherichia e Pseudomonas aeruginosa.

Segundo Melo (2009), das oito amostras estudadas de Staphylococcus aureus, sete destas com fenótipo de multirresistência foram inibidas com apenas $0,44 \pm 0,28$ $\mathrm{mg} / \mathrm{mL}$ do extrato aquoso de Syzygium malaccense. Este extrato apresentou maior sensibilidade frente às bactérias Gram-positivas principalmente Staphylococcus aureus. Resultados similares foram encontrados nesta pesquisa, onde o Staphylococcus aureus foi a única cepa que apresentou sensibilidade ao extrato de Syzygium malaccense.

$\mathrm{Na}$ análise dos dados dos extratos alcoólicos, Psidium guajava e Syzygium cumini demonstraram atividade antimicrobiana diante das quatro cepas estudadas. Sobre Strepctococcus pyogenes foi observado com halos de 10 $\mathrm{mm}$ e $11 \mathrm{~mm}$ respectivamente, na concentração de 100 $\mathrm{mg} / \mathrm{mL}$ cada.

Diante de Enterococcus faecalis foi observado halos de $8 \mathrm{~mm}$ e $11 \mathrm{~mm}$, respectivamente, na concentração de $100 \mathrm{mg} / \mathrm{mL}$. Apresentaram ainda, halos de inibição sobre Pseudomonas aeruginosa com $8 \mathrm{~mm}$ e $9 \mathrm{~mm}$, respectivamente, na concentração de $100 \mathrm{mg} / \mathrm{mL}$.

O extrato alcoólico de Syzygium malaccense neste estudo não apresentou atividade antimicrobiana frente às cepas estudadas. Vale e Orlanda (2011) ressaltam que, a ausência de atividade antimicrobiana pode ser devido à presença de baixas concentrações dos fitoconstituintes, 0 que acaba refletindo em baixa atividade da planta, pois as respectivas cepas mostraram-se resistentes aos extratos em todas as concentrações.

Gonçalves et al. (2008), em seus estudos in vitro com micro-organismos extraídos de camarão, observaram que o efeito inibitório melhor observado foi com extrato metanólico das folhas de Psidium guajava contra Staphylococcus aureus. Os halos de inibição variaram entre 8,25 e $9,25 \mathrm{~mm}$ sob a concentração estimada de 96,87 $\mu \mathrm{g} / \mathrm{mL}$. Pereira et al. (2010), testaram e comprovaram a ação antimicrobiana dos extratos glicólicos de Psidium guajava contra cepas padrão de Staphylococcus aureus.

Os resultados obtidos na atual pesquisas estão coerentes com o histórico da atividade antimicrobiana da espécie vegetal estudada, quanto à ação microbicida e à demonstração de que isolados das cepas estudadas mostraram-se sensíveis ao extrato alcoólico utilizado. É importante destacar que o extrato da Psidium guajava apresentaram atividade antimicrobiana frente às Gram-positivas (Staphylococcus aureus, Streptococcus pyogenes e Enterococcus faecalis) e Gram-negativas (Pseudomonas aeruginosa), as quais se diferem quanto à estrutura de sua membrana. Porém, é interessante ressaltar que a análise da atividade antimicrobiana nos diversos estudos citados foram realizadas por diferentes metodologias e concentrações, o que torna difícil uma análise comparativa fidedigna. 
Turatti (2008), analisando a ação antimicrobiana de Syzygium cumini, considerou as seguintes concentrações determinadas para a avaliação da atividade antimicrobiana de 78,12 a $2.500,0 \mu \mathrm{g} / \mathrm{mL}$. Utilizou os microrganismos Sthaphylococcus aureus, Sthaphylococcus epidermidis, Escherichia coli, Pseudomonas aeruginosa, Candida krusei, Candida albicans e Candida parapsilosis. O resultado de maior atividade na determinação da concentração inibitória mínima que ele obteve foi para os microrganismos Sthaphylococcus aureus, Sthaphylococus epidermidis e Candida parapsilosis, que se mostraram mais sensíveis aos extratos.

Os resultados obtidos, no presente trabalho, estão de acordo com dados da literatura, que indicam menor efetividade dos extratos de Syzygium cumini frente às bactérias Gram-negativas, como Pseudomonas aeruginosa, mesmo sendo sensíveis ao extrato alcoólico de Syzygium cumini, apresentaram halos menores se comparadas às Gram-positivas, como Sthaphylococcus aureus e Sreptococcus pyogenes.

As variações referentes à determinação da atividade antimicrobiana de extratos de plantas podem ser atreladas a muitos fatores. Diante deles, pode-se citar o método utilizado. De acordo com Pereira et al. (2010), essas técnicas e suas variantes devem ser adequados e escolhidos conforme as facilidades operacionais que concedam ensaios relativamente rápidos e simples, com menor custo. É importante que as adaptações sejam testadas e padronizadas para se obter reprodutibilidade e garantir, assim, que os resultados sejam confiáveis.

\section{CONCLUSÕES}

O presente estudo demonstrou que os extratos alcoólicos das espécies em estudo apresentaram melhor atividade antimicrobiana e maior espectro de ação quando comparados ao extrato aquoso e hidroalcoólico.

Através da técnica de poços por difusão em Ágar, os maiores halos de inibição ocorreram com os extratos hidroalcóolicos e aquosos de Eugenia uniflora frente à espécie de Staphylococcus aureus.

Todos os extratos das plantas estudadas apresentaram atividade diante de Staphylococcus aureus, exceto o extrato alcoólico de Syzygium malaccense.

O extrato alcoólico de Psidium guajava e Syzygium cumini apresentaram atividade contra todas às cepas utilizadas na pesquisa.

\section{REFERÊNCIAS}

ALVES, E. S.; TRESMONDI, F.; LONGUI, E. L. Análise estrutural de folhas de Eugenia uniflora L. (Myrtaceae) coletadas em ambientes rural e urbano, SP, Brasil. Acta Bot. Bras., São Paulo, v. 22, n. 1, p. 241-248, 2008.

ARAUJO, Y. L. F. M. et al. Comparação entre duas técnicas utilizadas no teste de sensibilidade antibacteriana do extrato hidroalcoólico de própolis vermelha. Scientia Plena, v. 7, n. 4, 2011.

AURICCHIO, M. T. et al. Atividades antimicrobiana e antioxidante e toxicidade de Eugenia uniflora. Acta farm. bonaer., Buenos Aires, v.
26, n. 1, p. 76, 2007.

BEZERRA, N. A. Avaliação da atividade antimibrobiana de Eugenia uniflora L. 2012. 37 f. Trabalho de Conclusão de Curso (Graduação em Ciências Biológicas) - Universidade Estadual da Paraíba, Paraíba, 2012.

BOUZADA, M. L. M. et al. Busca de novas drogas antimicrobianas a partir de vegetais. Principia - Caminhos da Iniciação Científica, Minas Gerais, v. 11, 8 p., 2007. Disponível em: <http://www.ufjf.br/principia/ edicoes-on-line/principia-caminhos-da-iniciacaocientifica-vol-9-2007>. Acesso em: 05 Nov. 2015

BRASIL. Ministério da Saúde. Políticas Nacionais de Plantas e Fitoterápicas. Brasília: Ministério da Saúde, 2006.

COSTA, J. G. M. et al. Estudo químico-biológico dos óleos essenciais de Hyptis martiusii, Lippia sidoides e Syzigium aromaticum frente às larvas do Aedes aegypti. Rev. Bras. Farmacogn., São Paulo,, v. 15, n. 4, p. 304-309, 2005.

ELLER, C.W.S. et al. Avaliação antimicrobiana de extratos vegetais e possível interação farmacológica in vitro. Rev. ciênc. farm., Araraquara, v.36, n.1, p.131-136, 2015.

FURTADO, J.M. et al. Atividade antimicrobiana do extrato aquoso de Eucalyptus globulus, Justicia pectoralis e Cymbopogon citratus frente a bactérias de interesse. UNOPAR Cient., Ciênc. Biol. saude, v.17, n.4, p.233-237, 2015.

GONÇALVES, F. A et al. Antibacterial activity of guava, Psidium guajava Linnaeus, leaf extracts on diarrhea-causing enteric bacteria isolated from Seabob Shrimp. Rev. Inst. Med. Trop. São Paulo, São Paulo, v. 50, n. 1, p. 11-15, 2008.

HOLETZ, F. B. et al. Screening of some plants used in the Brazilian folk medicine for the treatment of infectious diseases. Mem. Inst. Oswaldo Cruz, Rio de Janeiro, v. 97, n.7, p. 1027-1031, 2002.

LIMA, M. L. S. DE et al. Atividade antimicrobiana do jambolão (Syzygium cumini), ATRAVÉS DA TÉCNICA DE DIFUSÃO EM AGAR. Infarma, v. 23, n. $7 / 8$, p. 3-7, 2012.

LOPES, D. C. D. X. P. et al. Atividades antimicrobiana e fototóxica de extratos de frutos e raízes de Physalis angulata L. Rev. Bras. Farmacogn, São Paulo, v. 16, n. 2, p. 206-210, 2006.

LORENZI, H.; MATOS, F. J. A. Plantas medicinais no Brasil: nativas e exóticas cultivadas. São Paulo: Instituto Plantarum, 2008.

MELO, R. R. de. Perfil fitoquímico, avaliação da atividade antimicrobiana e biocompatibilidade de Syzygium malaccense (L) Merr. \& LM Perry (Myrtaceae). 2009. 74 f. Dissertação (Mestrado em Ciências Farmacêuticas) - Universidade Federal de Pernambuco, Recife, 2009.

MENEZES, M. DE C.; SOUZA, M. M. S. DE; BOTELHO, R. P. Avaliação in vitro da atividade antimicrobiana de extratos de plantas brasileiras sobre bactérias isoladas da cavidade oral de cães. Rev. Univ. Rural, Rio de Janeiro, v. 24, n. 2, p. 141-144, 2004.

MOREIRA, V. C.; FREIRE, D. Klebsiella pneumoniae e sua resistência a antibióticos. In: MOSTRA DE PRODUÇÃO CIENTÍFICA DA PÓS-GRADUAÇÃO LATU-SENSU DA PUC DE GOIÁS, 6., 2011, Goiás. Anais eletrônicos... Goiás: PUC, 2011. Disponível em: <http://www.cpgls.ucg.br/6mostra/ Artigos4.html>. Acesso em: 21 Nov. 2015.

OLIVEIRA, C. B.; SOARES, D. G. S.; PADILHA, W. W. N. Estudo in vivo sobre a eficácia da descontaminação de escovas dentárias pelo uso do spray de óleo essencial da Eugenia uniflora L. (Pitanga). In: REUNIÃO ANUAL DA SOCIEDADE BRASILEIRA DE PESQUISA ODONTOLÓGICA, 24., 2007, São Paulo. Anais... São Paulo: Brasilian Oral Research, 2007.

PAZHANI, G. P. et al. Clonal multidrug-resistant Shigelladysenteriae Type 
1 strains associated with epidemic and sporadic dysenteries in Eastern India. Antimicrob. Agents Chemother., Washington, v. 48, n. 2, p. 681684, 2004.

PEREIRA, A. V et al. Estudo comparativo dos extratos de Momordica charantia Linn. e Psidium guajava Linn. sobre linhagens de Staphylococcus aureus de origem bovinas isoladas no estado da Paraíba. Ciênc. agrotec., Lavras, v. 31, n. 2, p. 20-28, 2010.

SILVA, N. C. C. Estudo comparativo da ação antimicrobiana de extratos e óleos essenciais de plantas medicinais e sinergismo com drogas antimicrobianas. 2010. 75f. Dissertação (Mestrado em Biologia Geral e Aplicada) - Universidade Estadual Paulista. Botucatu, São Paulo, 2010.

SOBRAL, M. A família Myrtaceae no Rio Grande do Sul. São Leopoldo: Ed. Unisinos, 2003.

TAVEIRA, C. C. Ação antimicrobiana de extratos de plantas do Cerrado e isolamento de substância ativa de Kielmeyeracoriacea. 2007.
Dissertação (Pós - Graduação em Ciências Médicas) - Universidade de Brasília, Brasília, 2007.

TURATTI, K. F. M. Syzygium cumini (L.) Skeels: avaliação da qualidade, estudo morfo-anatômico, estudo da atividade antimicrobiana, conservante, genotóxica, mutagênica, citotóxica e incorporação em formulações cosméticas para uso tópico. 2008. 184 f. Tese (Doutorado) - Universidade Estadual Paulista, Faculdade de Ciências Farmacêuticas, São Paulo, 2008.

TYLER, V. E. Phytomedicines: black to the future. J. nat. prod., Cincinnati, v. 62, n. 3, p. 1589-1592, 1999.

VALE, V. V.; ORLANDA, J. F. F. Atividade antimicrobiana do extrato bruto etanólico das partes aéreas de Euphorbia tirucalli Linneau (Euphorbiaceae). Scientia Plena, v. 7, n. 4, 2011.

YUNES, R. A.; CALIXTO, J. B. (Eds.). Plantas medicinais sob a óptica da química medicinal moderna. Chapecó: Argos, 2001.
Submetido em: 22/09/2016

Aceito em: 24/04/2017 